Supporting Information

\title{
Synthesis of Few-Layer Graphene Sheets from Waste Expanded Polystyrene by Dense Fe Cluster Catalysis
}

Tianzhao Hu, Jiafu Chen*, Xiaoqing Lu, Jing Chen, Zhimin Chen, Jianwei Fu, Yong Chen

School of Materials Science and Engineering, Zhengzhou University, Zhengzhou, P.R. China, 450001

Email: chenjf@zzu.edu.cn

Results and discussion

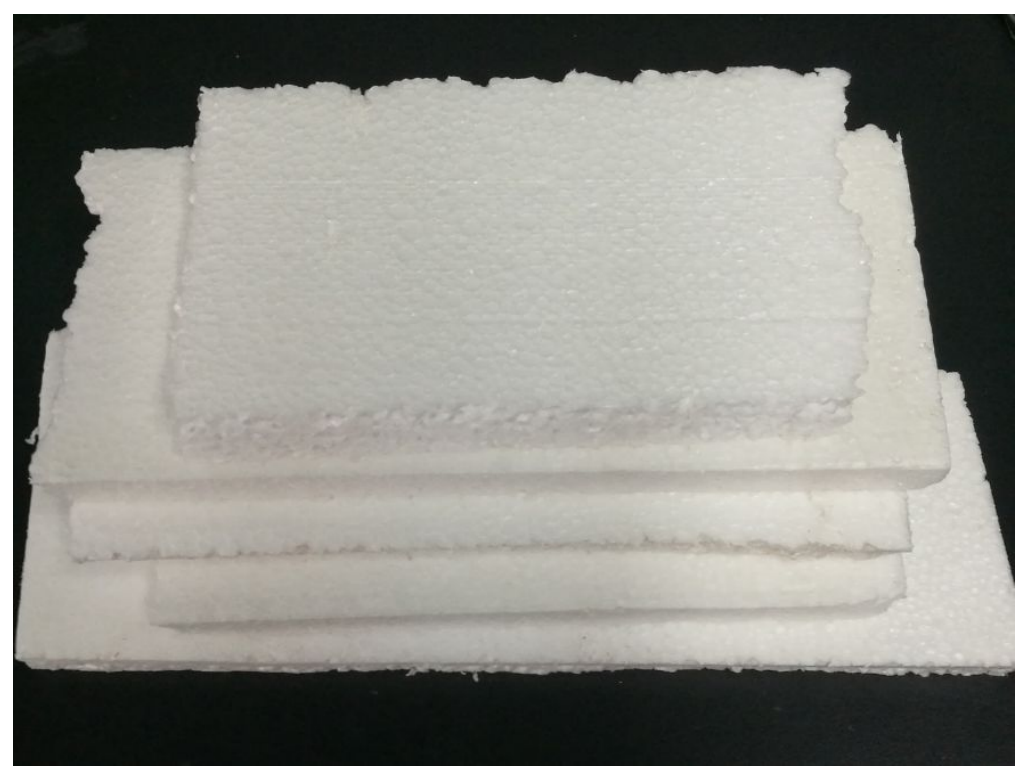

Figure S1. Digital image of waste expanded polystyrene (EPS) boards. 


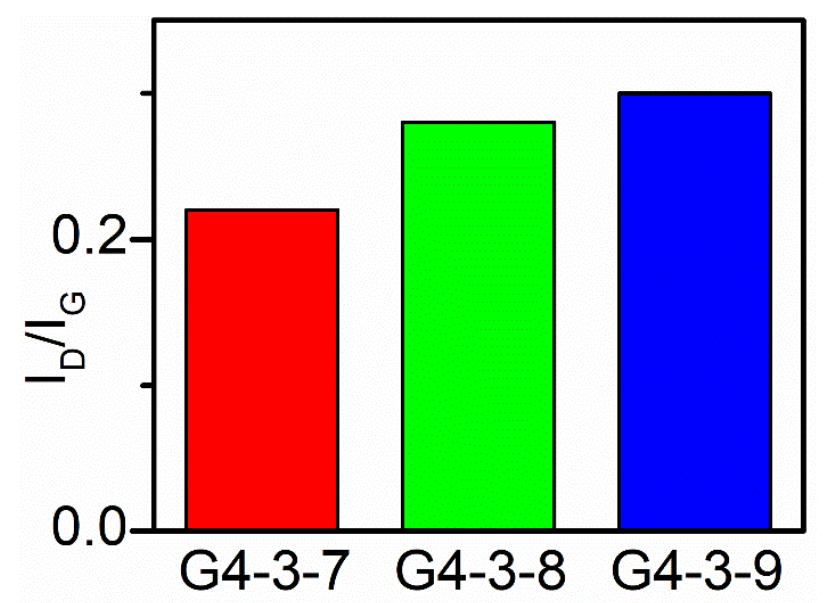

Figure S2. The $I_{\mathrm{D}} / I_{\mathrm{G}}$ values for G4-3-7, G4-3-8 and G4-3-9. 


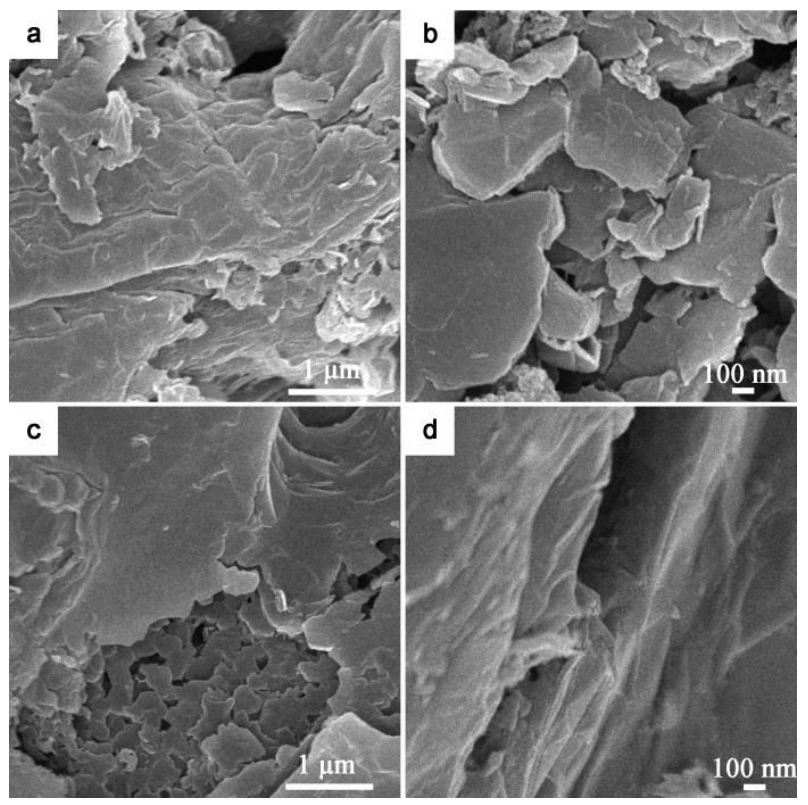

Figure S3. SEM images of (a, b) G4-3-7 and (c, d) G4-3-8. 

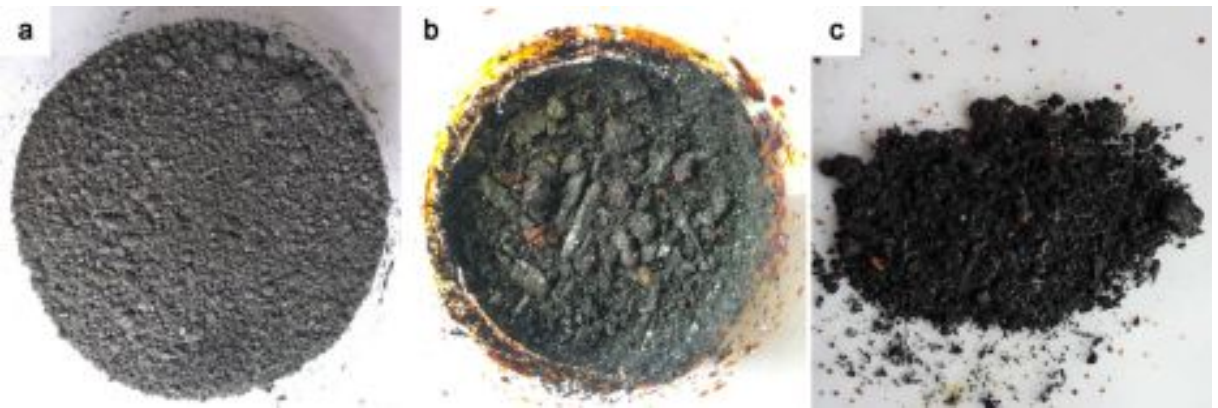

Figure S4. (a) Digital image of G4-3Fe, showing that after hypercrosslinking reaction, $\mathrm{FeCl}_{3}$ as catalyst became into grayish $\mathrm{FeCl}_{2}$ which loaded in hypercrosslinked EPS. (b, c) Digital image of the product obtained by stirring $\mathrm{FeCl}_{3}(3 \mathrm{~g})$ and DCE $(30 \mathrm{~mL})$ mixture at $60^{\circ}$ for 16 h, followed by evaporation of DCE, still indicating its own color of $\mathrm{FeCl}_{3}$. 


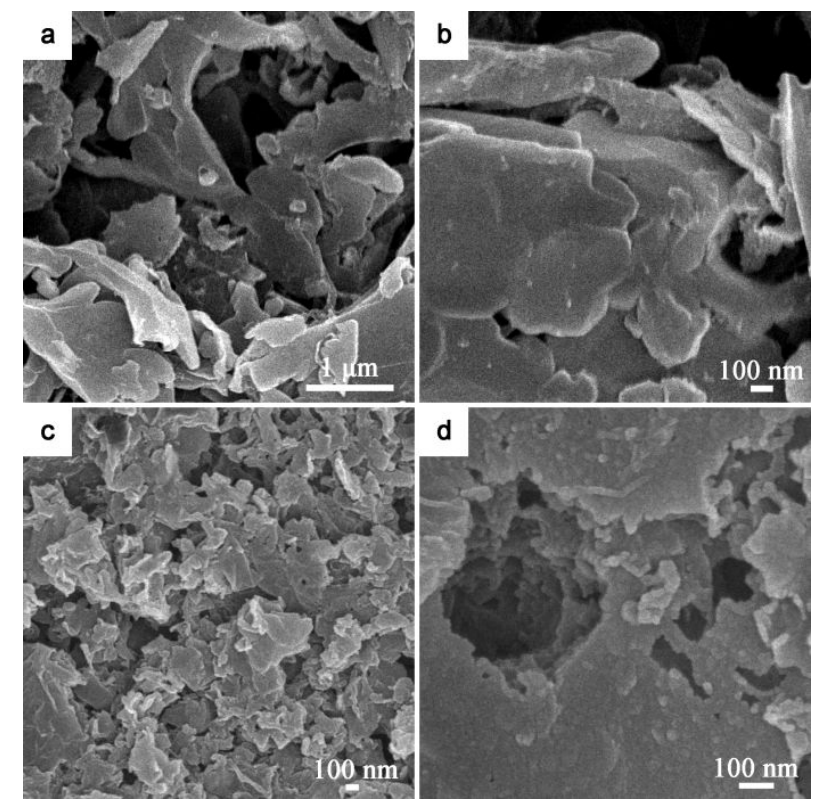

Figure S5. SEM images of (a, b) G4-10-9 and (c, d) G4-0.6-9. 


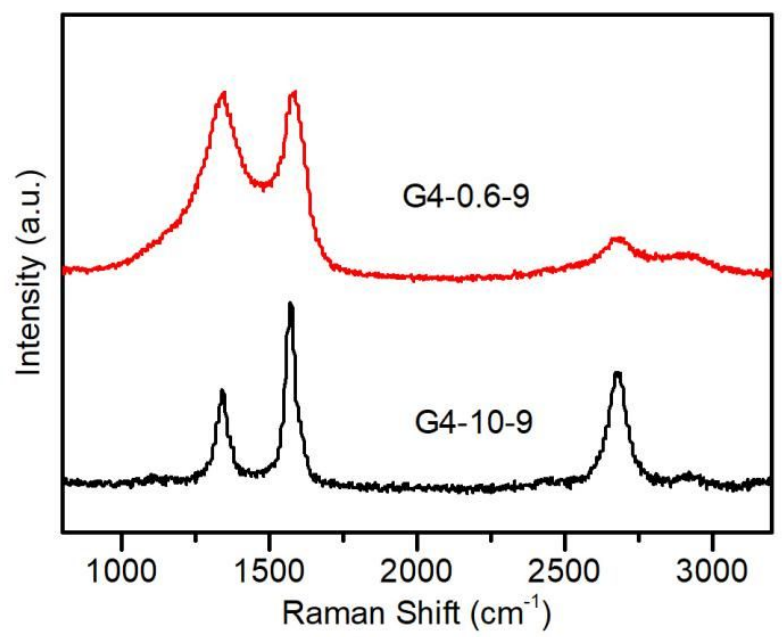

Figure S6. Raman spectra of G4-0.6-9 and G4-10-9. 

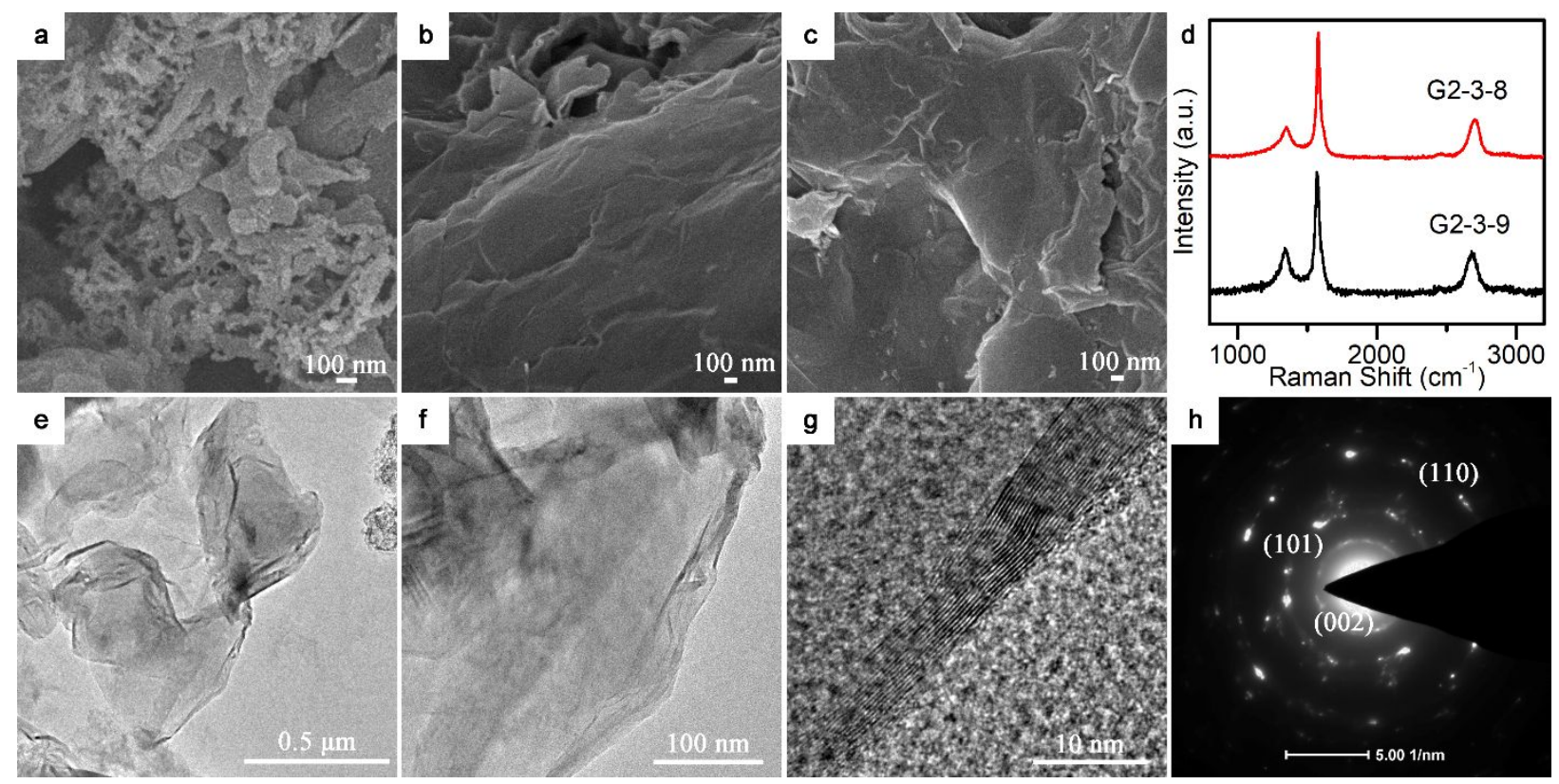

Figure S7. SEM images of (a) G0-3-9, (b) G2-3-8 and (c) G2-3-9. (d) Raman spectra of

G2-3-8 and G2-3-9. (e, f) TEM images, (g) HRTEM image and (h) corresponding SAED pattern of G2-3-8.

G0-3-9 is mainly messy amorphous carbon due to lack of pre-crosslinking step (Figure S7a). FLG sheets with high crystallinity were also successfully prepared at $800-900{ }^{\circ} \mathrm{C}$ by carbonizing $\mathrm{FeCl}_{2}$-loaded hypercrosslinked EPS with pre-crosslinking of $2 \mathrm{~h}$ (Figure S7b-g). It is suggested that pre-crosslinking is vital to obtain FLG sheets with high crystallinity. 